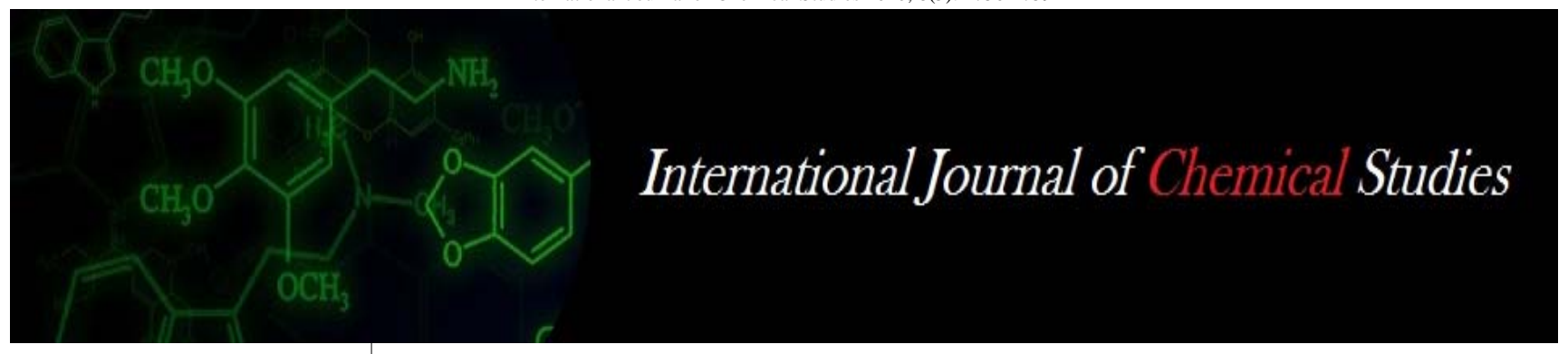

P-ISSN: 2349-8528

E-ISSN: 2321-4902 www.chemijournal.com IJCS 2020; 8(5): 1756-1759 (C) 2020 IJCS

Received: 16-07-2020

Accepted: 20-08-2020

Dr. Anil Kumar Singh Department of Chemistry Samastipur College Samastipur, Bihar, India

Corresponding Author: Dr. Anil Kumar Singh Department of Chemistry Samastipur College Samastipur, Bihar, India

\section{Supramolecular chemistry: Scope and perspective}

\author{
Dr. Anil Kumar Singh
}

DOI: https://doi.org/10.22271/chemi.2020.v8.i5x.10552

\begin{abstract}
Molecular chemistry is the chemistry of the covalent bond and concerned with the uncovering and mastering the rules that govern the structure, properties and transformation of molecules. Supramolecular chemistry may be defined as "Chemistry beyond the molecules". This is the chemistry of intermolecular bond covering the structure and functions of entities formed by association of two or more chemical species .Molecular recognition in the supramolecules formed by receptor-substrate binding .Molecular recognition is a process involving both binding and selection of substrate by a given receptor molecule.
\end{abstract}

Keywords: Supramolecular chemistry, molecular recognition, intermolecular bond, substrate, receptor, host, guest, entropic component

\section{Introduction}

Supramolecular chemistry is the chemistry of intermolecular bond, covering the structure and functions of the entities formed by association of two or more chemical species molecular recognition in the supramolecules formed by receptor-substrate binding rests on the principles of molecular complementarity, as found in spherical and tetrahedral recognitions, anion coordination. supramolecular catalysis by receptors bearing reactive groups effects bond cleavage reactions as well as synthetic, bond formation via catalysis lipophilic receptor molecules act as selective carriers for various substrates and allow to set up coupled transport processes linked to electron and proton gradients or to light whereas endo -receptors bind substrates in molecular cavities by convergent interactions, exo-receptors rely on interactions between the surface of receptors and the substrate; thus new type of receptors such as the metallo nucleates may be designed. In combination with polymolecular assemblies, receptors, carries and catalysts may lead to molecular and supramolecular devices, defined as structurally organized and functionally integrated chemical systems built on supramolecular architectures. There recognition, transfer and transformation features are analyzed specifically from the point of view of molecular devices that would operate via photons, electronor ions, thus defining fields of molecular photonics, electronics and ionics. Introduction of photosensitive groups yields photoactive receptors for design of light conversion and charge separation centres. Redox active polyolelinic chains represent molecular wires for electron transfer through membranes. Tubular mesophases formed by stacking of suitable macrocyclic receptors may lead to ion channels molecular self assembling occurs with acyclic ligands that form complexes of double helical structure. Such developments in molecular and supramolecular design and engineering open perspectives towards the realization of molecular photonic, electronic and ionic devices, that would perform highly selective recognition, reaction and transfer operations for signal and information processing at the molecular level.

Molecular chemistry, the chemistry of the covalent bond is concerned with uncovering and mastering the rules that govern the structures, properties and transformation of molecular species. supramolecular Chemistry may be defined as "chemistry beyond the molecule" bearing on the organized entities of higher complexity that results from the Association of two or more chemical species held Together by intermolecular forces its development require the use of all the forces of molecular chemistry combined with the designed manipulation of noncovalent interactions so as to form supramolecular entities, supermolecules possessing features as well declined as those of molecules themselves. One may say that supermolecules are to molecules and the intermolecular bond what molecules are to atoms and the covalent bond. 
supermolecules, was introduced already in the mid-1930's to describe entities of higher organization resulting from the association of coordinatively saturated species. The partners of a supramolecular species have been named molecular receptor and substrate, the substrate being usually the smaller component whose binding is being sought. This terminology conveys the relation to biological receptors and substrates for which Paul Ehrlich stated that molecules do not act if they are not bound. Molecular interactions form the basis of the highly specific recognition, reaction, transport, regulation etc. processes that occur in biology such as substrate binding to a receptor protein, enzymatic reactions, assembling of proteinprotein complexes, immunological antigen-antibody association, intermolecular reading, translation and transcription of the genetic code, signal induction by neurotransmitters, cellular recognition, etc. The design of artificial, abiotic, receptor molecules capable of displaying processes of highest efficiency and selectivity requires the correct manipulation of the energetic and stereochemical features of the non-covalent, intermolecular forces (electrostatic interactions, hydrogen bonding, Van der Waals forces etc.) within a defined molecular architecture. In doing so, the chemist may find inspiration in the ingenuity of biological events and encouragement in their demonstration that such high efficiencies, selectivities and rates can indeed be attained. However chemistry is not limited to systems similar to those found in biology, but is free to invent novel species and processes. Binding of a substrate $u$ to its receptor $\mathrm{Q}$ yields the supermolecule and involves a molecular recognition process. If, in addition to binding sites, the receptor also bears reactive functions it may effect a chemical transformation on the bound substrate, thus behaving as a supramolecular reagent or catalyst. A lipophilic, membrane soluble receptor may act as a carrier effecting the translocation of the bound substrate. Thus, molecular recognition, transformation and translocation represent the basic functions of supramolecular species. The present text describes these various aspects of supramolecular chemistry (diagrammatically shown in Scheme 1)

\section{CHEMISTRY
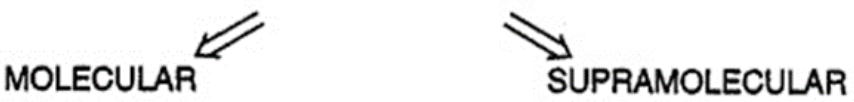

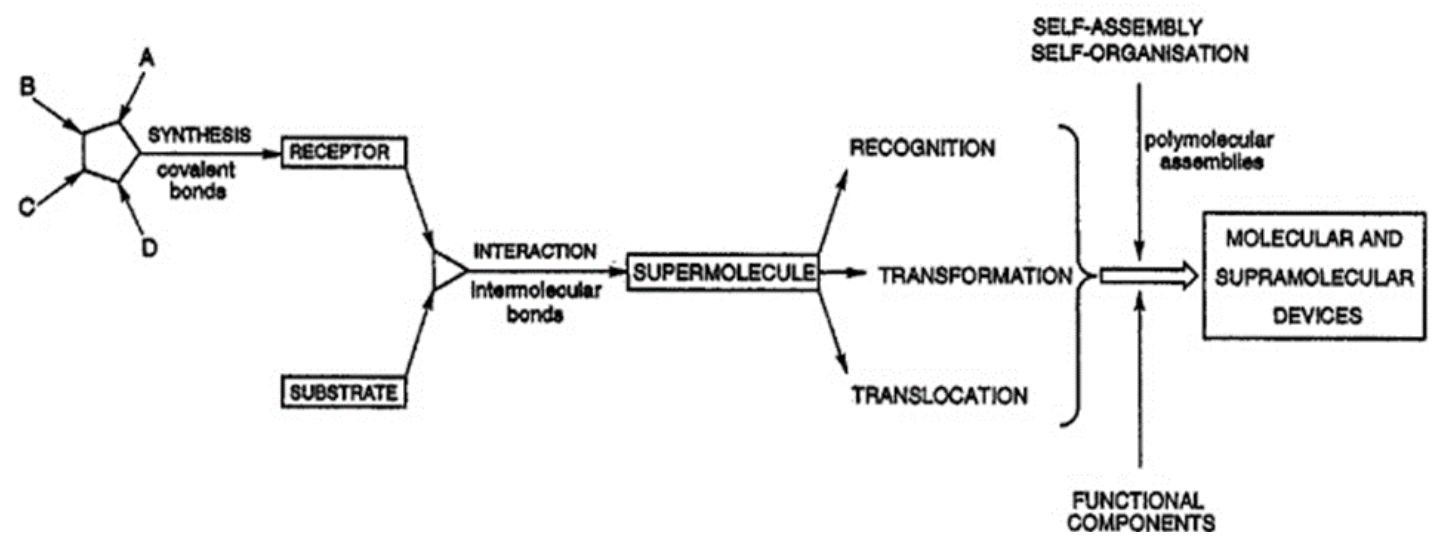

Scheme 1: From molecular to supramolecular chemistry: molecules super molecules, molecular and supramolecular devices.

Emphasis will bear on conceptual framework, classes of compounds and types of processes. Considering the vast literature that has developed, the topics of various meetings and symposia, etc., there is no possibility here to do justice to the numerous results obtained, all the more to provide an exhaustive account of this field of science. Supramolecular chemistry, the designed chemistry of the intermolecular bond, is rapidly expanding at the frontiers of molecular science with physical and biological phenomena.

\section{Molecular Recognition}

Molecular recognition has been defined as a process involving both binding and selection of substrate(s) by a given receptor molecule, as well as possibly a specific function. Mere binding is not recognition, although it is often taken as such. One may say that recognition is binding with a purpose, like receptors are ligands with a purpose. It implies a structurally well-defined pattern of intermolecular interactions.

The ideas of molecular recognition and of receptor chemistry have been penetrating chemistry more and more over the last fifteen years, namely in view of its bioorganic implications, but more generally for its significance in intermolecular chemistry and in chemical selectivity as shown in following boxes.

\section{Host-Guest chemistry}

Definition

Host: Organic molecule containing convergent binding sites. Synthetic counterpart to receptors sites in enzymes, genes, Antibodies and ionospheres 
Guest: Molecules or ions containing divergent binding sites. Counterparts to substrates, inhibitors, cofactors, antigens

Complexes: Host and guests held together in solution in definable structural Relationship by electrostatic force (enthalpic component) such as Ion pairing, hydrogen bonding, metal ion-to-ligand attraction, $\pi-\pi$ Stacking, dipole- dipole interactions, and van der waals attraction, And the entropic component of desolvation

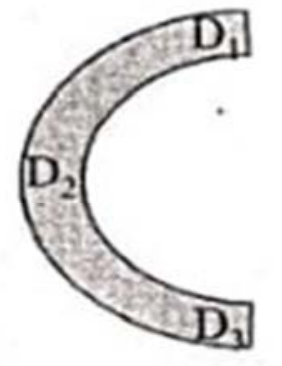

Host

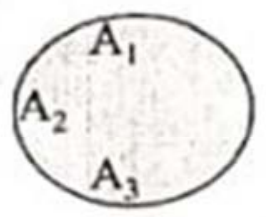

Guest

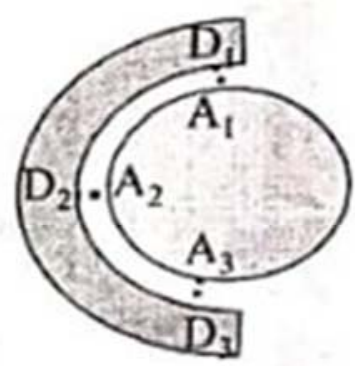

Complex

\section{Examples of Synthetic Host Types}

\section{Macrocyclic}

Clefts

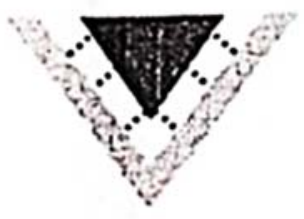<smiles>C1COCCOCCOCCOCCO1</smiles>

Crown Ether

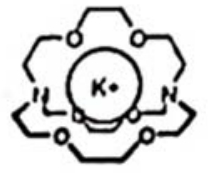

Cryptand
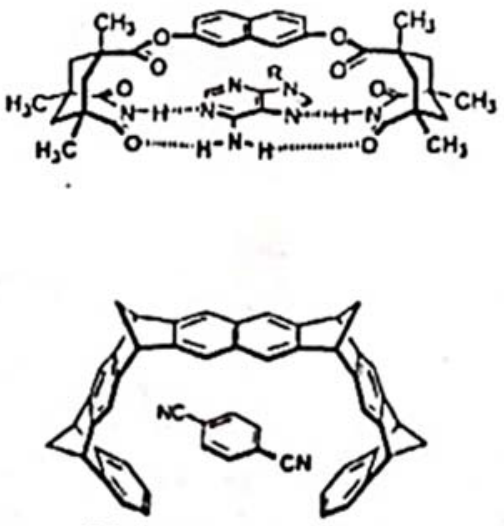

Molecular Tweezer
Bowls
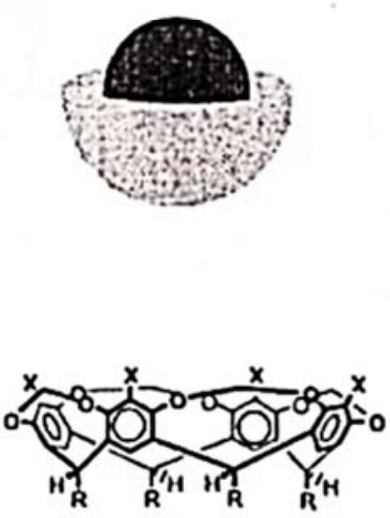

Cavitand

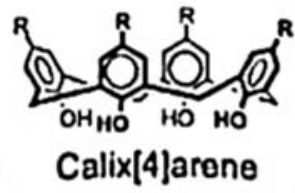

\section{Linear}

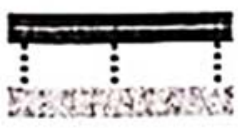

\section{Conclusion}

The present text was aimed at presenting the scope, providing illustration and exploring perspectives of supramolecular chemistry. Its conceptual framework has been progressively laid down and the very active research on molecular surfaces and polynuclear assemblies, is building up a vast body of knowledge on molecular behaviour at the supramolecular level. It is clear that much basic chemistry remains to be done on the design and realization of numerous other systems and processes that await to be imagined. The result obtained may also be analysed in the view of developing components for molecular devices that would perform highly selective functions of recognition, transformation, transfer, regulation and communication, and allow signal and information processing. This implies operation via intermolecular interactions and incorporation of the time dimension into recognition events. One may note that such functions have analogies with features of expert systems, thus linking processes of artificial intelligence and molecular behaviour. These developments in molecular and supramolecular science and engineering offer exciting perspectives at the frontiers of chemistry with physics and biology. Of course even the past 
achievements and present activities, extrapolation and predictions can only be tentative; yet, on such an occasions for the celebration of chemistry, it appears justified to try looking out into the future for "He (or she) who sits at the bottom of a well to contemplate the sky, will find it small”

\section{Acknowledgements}

I wish to thanks very warmly my research supervisor Dr. Bigan Ram, associate professor, department of chemistry, Women`s College, Samastipur whose skill, dedication and enthusiasm, allowed the work described here to be realized.

\section{References}

1. Lehn, JM. Supramolecular chemistry and selforganisation, Proc. Natl. Acad. Sci., 2002; 99:4763.

2. Lehn JM. Supramolecular chemistry towards constitutional dynamic.

3. Chemistry and adaptive chemistry, Chem. Soc. Rev., 2007; 36:151. 